Henning, Günther; Wiechmann, Volker:

\title{
Ophthalmoinnovation Thüringen
}

\author{
Zuerst erschienen in: Biomedizinische Technik = Biomedical Engineering. - Berlin [u.a.] : \\ de Gruyter. - 47 (2002), S1b, S. 890-892. \\ Jahrestagung der Deutschen Gesellschaft für Biomedizinische \\ Technik (DGBM) im VDE ; 36 (Karlsruhe) : 2002.09.25-27 \\ Erstveröffentlichung: 2002 \\ Datum Digitalisierung: $2009-11-26$ \\ ISSN (online): $\quad$ 1862-278X \\ ISSN(print) 0013-5585 \\ DOI: $\quad$ 10.1515/bmte.2002.47.s1b.890 \\ [Zuletzt gesehen: 2019-12-12]
}

„Im Rahmen der hochschulweiten Open-Access-Strategie für die Zweitveröffentlichung identifiziert durch die Universitätsbibliothek IImenau."

"Within the academic Open Access Strategy identified for deposition by IImenau University Library."

„Dieser Beitrag ist mit Zustimmung des Rechteinhabers aufgrund einer (DFGgeförderten) Allianz- bzw. Nationallizenz frei zugänglich."

„This publication is with permission of the rights owner freely accessible due to an Alliance licence and a national licence (funded by the DFG, German

Research Foundation) respectively."

\section{DFG}

Nationallizenzen 


\title{
OPHTHALMOINNOVATION THÜRINGEN
}

\author{
G. Henning ${ }^{1}$, V. Wiechmann ${ }^{2}$ \\ ${ }^{1}$ Institut für Biomedizinische Technik und Informatik, Technische Universität Ilmenau, Deutschland \\ ${ }^{2}$ OphthalmoInnovation Thüringen, Jena, Deutschland \\ guenter.henning@tu-ilmenau.de
}

\begin{abstract}
OphthalmoInnovation Thüringen is focused on the development of innovative products and services for ophthalmology with the following main points of emphasis: Systems for objective functional diagnostics of vision, metabolism and microcirculation of the eye, therapeutic systems for laser applications in ophthalmology. Current research projects are oriented to functional imaging of to retinal microcirculation, to objective perimetry, to optimising refractive laser surgery and to photodynamic therapy methods. - The very open cooperation between research institutions and companies in the OIT network ensures an effective way from a research result to an innovative product.
\end{abstract}

Keywords_ophthalmology, functional imaging, objective perimetry, laser therapy

\section{Ziele}

Inhaltliche Zielstellung des Kompetenzzentrums ist die Neu- und Weiterentwicklung innovativer Produkte und Dienstleistungen für die Ophthalmologie mit folgenden Schwerpunkten:

- Funktionsdiagnostische Systeme zur objektiven Beurteilung der Funktionen des Sehvorganges, des Stoffwechsels und der Mikrozirkulation des Auges

- Therapeutische Systeme für die Laseranwendung in der Augenheilkunde.

Damit sollen Beiträge geleistet werden zur Entwicklung von neuartigen Gerätesystemen für die Bekämpfung von Erblindungsursachen durch Risiko-Screening, Früherkennung, individuelle Therapie-Optimierung und für die medizinische Qualitätskontrolle mit dem Ziel der Kostensenkung und Qualitätsverbesserung im Gesundheitswesen.

Die strategischen Ziele sind:

- Sicherung und Ausbau internationaler Wettbewerbsfähigkeit der einbezogenen Industrieunternehmen und der beteiligten wissenschaftlichtechnischen Einrichtungen

- Förderung von Existenzgründungen und deren Unterstützung bei der Vermarktung

- Ausbau vorhandener wissenschaflicher und industrieller medizintechnischer Kompetenz in Thüringen und deren synergetische Vernetzung.

\section{Inhaltliche Schwerpunkte}

Diese Ziele werden zunächst in sieben Projektlinien umgesetzt, die sich durch eine entsprechende Innovationshöhe auszeichnen und in ihrer inhaltlichen Verknüpfung ein homogenes Profil bilden:
- Bildgebende Basistechnologien für Funktionsimaging und individuelle Therapieführung am Auge

- Funktionsimaging der Mikrozirkulation für die individuelle Therapieführung

- Spektralimaging zur Erfassung therapeutisch relevanter Kenngrößen des Stoffwechsels

- Elektrodiagnostik zur objektiven Beurteilung des visuellen Funktionszustandes

- Refraktive Laserchirurgie: Topografie- und Wellenfrontanalyse-gestützte Laserablation der Cornea mit online-Kontrolle

- Lasertherapeutische Kataraktbehandlung; LaserMikrochirurgie am Auge

- Neue Konzepte der Lasertherapie: MikrosekundenLaser, Photodynamische Therapie.

Die Auswahl dieser Projektlinien ist durch die möglichen Synergieeffekte in diagnostischer bzw. therapeutischer, gerätetechnischer und marktbezogener Relevanz im Sinne der Zielsetzung bestimmt. So ist der therapeutische Erfolg durch Medikation oder Laserbehandlung maßgeblich von den Therapiekriterien, der Indikation bzw. Kontraindikation, dem Behandlungsort, der Dosis und Wirkungsbeziehung determiniert.

Die hierzu notwendigen Informationen ergeben sich aus der gestörten Funktion der abgebildeten Augenabschnitte, der Mikrozirkulation, des Stoffwechsels und der objektiven Sehfunktion. Die funktionsdiagnostischen Methoden sind Ausgangspunkt und liefern zugleich Bewertungskriterien für die Effizienz der Therapie. Methoden der künstlichen Intelligenz, insbesondere der Modellbildung, sollen eine kausal orientierte effektive und individuelle Therapie stützen und die komplexen Zusammenhänge zwischen den verschiedenen Kenngrößen der Funktionsdiagnostik untereinander zur Erreichung des therapeutischen Erfolgs nutzbar machen.

\section{Ausgewählte Projektlinien}

Im folgenden sollen beispiclhaft 3 aktuelle Projektlinien mit ihren Zielstellungen, Lösungsansätzen und ersten Ergebnissen vorgestellt werden.

Spectral Imaging: Die Haupterblindungsursachen in den entwickelten Industrieländern sind Glaukom. altersbedingte Makuladegeneration (AMD) und diabetische Retinopathic. Der zugrundeliegende Pathomechanismus dieser Erkrankungen ist weitgehend unbekannt. Dic bekannten therapcutischen Möglichkciten sind cinge. schränkt und berichen sich nur auf dic Bchandlung von Symptomen. Neuartige Therapicansätze sind nur dianı zu crwarten, wenn es gelingt, die Erkrankung in cincul 
möglichst frühen Stadium zu diagnostizieren, in $\mathrm{dem}$ der gestörtc Stoffwechselzustand sich noch nicht in irreversiblen morphologischen Verïnderungen manifesticrt hat.

7.icl des Verbundprojektes ist es, Anordnungen für ncue Melhoden der Frühdiagnostik von Stoffwechselerkrankungen am Augenhintergrund, spezicll von diabetischer Retinopathic und AMD, zu entwickeln, aufzubauen, zu verifizicren, Methodenvergleiche durchzuführen und bezïglich ihrer klinischen Wertigkeit zu evaluieren.

Ein tvesentlicher Schwerpunkt des Vorhabens ist die Entwicklung neuer Verfahren zur Gewinnung und Auswertung monochromatischer Fundusbilder, insbesondere zur 2-dimensionalen Bestimmung der Sauerstoffsättigung und der Verteilung des Makulapigments. Zur Bestimmung des Stoffwechselzustandes auf zellulärer Ebene sind die Grundsatzuntersuchungen auf die Realisierbarkcit und Interpretation von Bildern der zeitaufgelösten Autofluoreszenz des lebenden Patientenauges gerichtet.

Für beide Aufgaben werden in Kooperation mit Carl Zeiss Meditec Jena Demonstratoren realisiert. Nach theoretischen Abschätzungen und experimentellen Prinzipversuchen wurden die Hardware und die Software für die Demonstratoren aufgebaut und durch Tests erprobt. Wesentlich ist, dass sowohl für die Erzeugung monochromatischer Bilder als auch für die zeitaufgelöste Messung der Autofluoreszenz in der Ophthalmologie neuartige Funktionsprinzipien eingesetzt werden, deren Realisierbarkeit und Ergebnisse unter den Bedingungen der maximal zulässigen Exposition des lebenden Auges noch bewiesen bzw. bewertet werden müssen.

Zur Konzeptionierung, Entwicklung und Erprobung des „Polarisations-Anisotropie-Imager PAI“ (eines Systems zur 2-dimensionalen Erfassung des Sauerstoffpartialdrucks am Augenhintergrund.) bei Carl Zeiss Meditec (CZM) erfolgt eine enge Kooperation mit der UAK der FSU Jena. Für die Verifikation dieser neuen Methode werden geeignete Testanordnungen entwickelt. Danach erfolgt an der UAK unter Mitwirkung von CZM der Methodenvergleich mit den dort realisierten Methoden zur 2-dimensionalen Erfassung von Sauerstoffsättigung, optischer Dichte von Pigmenten und Autofluoreszenz sowie eine erste klinische Erprobung. Die klinische Evaluierung in weiteren Referenzkliniken wird über das Projektende ausgedehnt.

Elektrodiagnostik des visuellen Systems: In der Funktionsdiagnostik des visuellen Systems ist eine Objektivierung der Aussage über die Reizwahrnehmung oft notwendig und sinnvoll. Die medizinische Indikation ergibt sich aus der häufig fehlenden Kooperation des Patienten. Diese besteht im wesentlichen aus der Bereitschaft, den Blick auf einen definierten Punkt der Perimeterkugel zu fixieren und bei Reizwahrnehmung dies entsprechend - z.B. durch Knopfdruck oder Meldung - zu signalisieren. Ziel dieses Projektes ist es, das Problem der fehlenden Kooperation aus der Sicht der Stimulation und der Detektion der Reizwahmehmung aus einem objektiven Messsignal (VECP) zu lösen.
Der Ansatz zur Blickrichtungsbestimmung kann den VOG-Verfahren (Videookulografie) zugeordnet werden. Dic bisherigen VOG-Verfahren weisen wesentliche Nachteile auf. Es wurde daher ein neues VOG-Verfahren entwickelt, das kalibrationsfrci arbeitet und Kopfbewegungen im Zentimetcrbercich toleriert. Dieses Verfahren basiert auf dem Prinzip der stcrcoskopischen Vermessung. Zum Erreichen cincr akzeptablen Untersuchungszeit in der Größcnordnung der subjcktiven Verfahren ist die Entwicklung neuer Stimulationsfolgen (Multifokale Stimulation) und Detektionsmethoden für die VECP-Signale notwendig. Im bisherigen Projektablauf konnten neuartige Lösungsansätze multifokaler Reizung erstellt und getestet werden. Auch auf dem Gebiet der Detektion der Reizantworten am visuellen Kortex wurden Fortschritte in der Entwicklung adaptiver Fokussierungsalgorithmen erreicht. Bisher wurden die neuen Methoden an Modellen und zum Teil an realen Daten getestet. Alle Ansätze konnten bestätigt werden. In der Blickrichtungsbestimmung wurde die geforderte Genauigkeit und Geschwindigkeit erreicht. In der Stimulation wurden neue multifokale Folgen an synthetischen Daten evaluiert, Messungen an Probanden werden gegenwärtig durchgeführt. Für die Detektion der Reizantwort konnte mit Hilfe neuer Beamingmethoden die erforderliche Verbesserung des Signal-Rausch-Abstandes im Mittel erreicht werden.

Eine direkte Übertragung der Ergebnisse vom Modell auf reale Verhältnisse ist nicht sinnvoll. Eine systematische Evaluierung ist aber kaum möglich, da weder die genaue Blickrichtung bei realen Augen hinreichend genau bestimmt werden kann, noch lässt sich der tatsächliche Signal-Rausch-Abstand im realen EEG zuverlässig abschätzen. Ausgehend davon, dass die Modellansätze die Anforderungen erfüllen und damit besser als bisherige Verfahren sind, kann jedoch mit einer deutlichen Verbesserung auch im realen Messaufbau gerechnet werden.

Visusoptimierende refraktive Laserchirurgie: Die refraktive Laserchirurgie (RLC) mittels Excimer-Laser hat sich als eine Methode der Wahl zur Korrektur typischer Visusdefekte wie Myopie, Hyperopie und Astigmatismus etabliert. Mit den Standardverfahren können allerdings bis heute Aberrationen höherer Ordnung nicht korrigiert werden. Es ist das Ziel dieses Projekts, ein Verfahren zur präzisen messtechnischen Bestimmung dieser Aberrationen als entscheidende Voraussetzung zu ihrer Korrektur sowie einen Demonstrator zum Nachweis der Wirksamkeit und Reproduzierbarkeit der Korrektur mittels RLC zu entwickeln.

In Voruntersuchungen wurde als optimal geeignete Messmethode die Wellenfrontanalyse auf der Basis eines ShackHartmann-Sensor-basierten Systems ausgewählt. Dieses System weist ein außerordentlich gutes Verhältnis zwischen Realisierungsaufwand, Kosten und Verfügbarkeit auf. Gegenüber seriellen ,ray tracing“-Methoden, bei denen die Wellenfront Punkt für Punkt abgetastet wird, können Shack-Hartmann basierte Systeme die gesamte Wellenfront in einem Schritt messen, was bei schnell veränderlichen biologischen Systemen (Augenbewegungen) von besonderem Vorteil ist. Die methodische Lösung wurde bereits in einem neuen Produkt (WASCA 
Wavefront Analyzer) umgesetzt. Die Kopplung zum Ablationslaser wurde realisiert; die Ablationsalgorithmen wurden entwickelt. Eine erste umfassende $\mathrm{Be}$ wertung des Messsystems mittels Untersuchungen am Modell (Testaugen, Aberrations-Phasenplatten) bestätigte die erwarteten exzellenten Parameter. In einer weiteren Modelluntersuchung - ebenfalls an Phasenplatten - wurde der Nachweis der erforderlichen Genauigkeit der Ablation geführt. Auf Basis der Ergebnisse einer in-vitro-Studie konnten die Ablationsalgorithmen optimiert werden.

Neben den Ergebnissen der in vitro-Studien ist die Kenntnis der genauen physiologischen Augendaten und des Augenaufbaus (Augenmodell) für die Korrektur des Visus in vivo unabdingbar, da insbesondere die Korrekturen höherer Aberrationen empfindlich auf die physiologischen Augendaten reagieren, was auf die geringen Wellenfronthübe (und damit Ablationstiefen) und die hohe räumliche Modulation dieser Aberrationen zurückzuführen ist. Im Ergebnis einer Studie zum Entwurf eines schematischen Augenmodells zur Simulation der Auswirkungen verschiedener Ablationsformen bzw. Ablationsbeiträge bei der refraktiven Visuskorrektur konnte festgestellt werden, dass der möglichst korrekten Wiedergabe des physiologischen Aufbaus und der möglichst realen Simulation der optischen Parameter und Abbildungseigenschaften des menschlichen Auges (nach jetzigem Kenntnisstand) das Liou-BrennanAugenmodell am besten entspricht.

International wird die Relevanz der Diagnose höherer Aberrationen für ein tieferes Verständnis und die Verbesserung der heutigen Operationstechnik für Visuskorrekturen intensiv diskutiert und unterstrichen; es hat eine rasante Entwicklung eingesetzt. Mit dem beschriebenen Stand der Forschung hat der OIT-Partner Zeiss Meditec eine solide Basis für eine erfolgreiche Produktinnovation geschaffen.

\section{Perspektive des Zentrums}

Das Technologiedreieck Jena-Ilmenau-Erfurt besitzt traditionell eine bedeutsame WissenschaftsWirtschaftsstruktur, die in geradezu idealer Weise durch ausgezeichnete medizinische Lehre, Forschung und Betreuung ergänzt wird. Namhafte Hersteller von Produkten der Medizintechnik für die Ophthalmologie sind hier beheimatet. Die im OIT-Netzwerk verbundenen Partner bilden eine durchgängige Kompetenzkette von der medizintechnischen und medizinischen Forschung über die klinische Evaluierung und Applikation bis zur industriellen Entwicklung und Umsetzung. Durch das Kompetenzzentrum wird die in der Region vorhandene hervorragende Infrastruktur noch stärker auf Erfordernisse des Ophthalmologiemarktes ausgerichtet. - Langfristige Finanzierungsstrategien für die Zentrumskoordination gewährleisten die Nachhaltigkeit des Konzepts über den Förderzeitraum hinaus.

\section{Danksagungen}

Ohne eine stabile Förderung über einen mittelfristigen Zeitraum wären der Aufbau eines leistungsfähigen Kompetenzzentrums, die Entwicklung innovativer Schwerpunktlinien und die Umsetzung eines auf Nachhaltigkeit orientierten Konzepts nicht möglich. Für diese Förderung danken wir dem Bundesministerium für Bildung und Forschung, dem Thüringer Ministerium für Wissenschaf, Forschung und Kunst, dem Thüringer Ministerium für Wirtschaft, Arbeit und Infrastruktur sowie der Stiftung für Technologie- und Innovationsförderung Thüringen. Für eine intensive kritische Begleitung sind wir dem Projektträger VDI-TZ Düsseldorf zu Dank verpflichtet. 